\title{
THE INCIDENCE OF EPISTASIS IN NORMAL AND EXTREME ENVIRONMENTS
}

\author{
J. L. JINKS, JEAN M. PERKINS and H. S. POONI \\ Department of Genetics, University of Birmingham, Birmingham B15 2TT, England
}

Received 11.xi.72

\section{SUMmary}

To account for the genotype-environmental interactions which occur between families derived from crosses between the true-breeding varieties 1 and 5 and 2 and 42 of Nicotiana rustica and the environmental variables, sowing date and planting density, it is necessary to include interactions between epistatic gene action and these environmental variables for most characters. In general the epistatic component of the family means is greatest in one or both extremes of the environmental range and is smallest in the other extreme or in the average environments. The change in magnitude and sign of the epistatic component over environments in some but not in all cases is linearly related to biological or physical measures of the differences between the environments.

The same character may, therefore, display two or more distinct kinds of genetical architecture in different parts of the environmental range. In average environments most characters display the architecture of a character with an intermediate optimum which has been subjected to stabilising selection while in an extreme environment they display the architecture of a character with an optimum at one extreme of the phenotypic range which has been subjected to directional selection. This is as expected, provided that the type of selection to which a phenotype, which deviates from the optimum as defined in an average environment, is subjected, is the same irrespective of whether it is the product of an extreme genotype in an average environment or an average genotype in an extreme environment.

\section{INTRODUCTION}

IN our last paper (Perkins and Jinks, 1973) it was necessary to consider epistatic gene action and its interaction with the environment in order to interpret the interaction between 82 inbred lines, derived from a cross between pure-breeding varieties 1 and 5 of Nicotiana rustica, and eight combinations of two environmental variables, sowing dates and planting densities. In this paper we shall examine the relationship between the occurrence of epistasis and the kinds of environments in more detail.

\section{Material}

The analysis of two sets of data will be presented. The first set has been described and analysed by Perkins and Jinks (1973) and is the 82 inbred lines grown in eight environments, mentioned earlier. The second set of data consists of the pure-breeding varieties 2 and 42 and their $F_{1}, F_{2}$, and first backcross $\left(B_{1}\right.$ and $B_{2}$ ) generations grown in each of eight environments which consist of eight different sowing dates in the same season (Perkins and Jinks, 1971).

\section{Method}

The analysis of both sets of data has followed the same pattern. The environments within each set have been assessed and ranked on the mean 
performance of the pure-breeding parental varieties. In each environment the adequacy of a model specifying the generation means which assumes no epistasis has been tested. For the first set of data this test consists of a comparison between the mean of all inbred lines and that of the parental varieties, 1 and 5, which are expected to be the same in the absence of epistasis. The difference between the two means is a direct estimate of the epistatic component of the mean, [i] (Perkins and Jinks, 1973). For the other set of data the standard joint scaling test (Mather and Jinks, 1971) can be used.

\section{The incidence of EPISTASIS IN DIFFERENT ENVIRONMENTS}

In table 1 the estimates of [i] and their significances are listed in order of increasing environmental values for the eight environments of the first set of

\section{TABLE 1}

Estimates of the environmental value and of the epistatic component, [i], and the significance of the latter for each of eight environments for three characters of the cross $1 \times 5$

\section{Final height}

Environmental value

$85 \cdot 62$

$103 \cdot 12$

$103 \cdot 68$

$105 \cdot 31$

$116 \cdot 50$

117.50

124.68

$128 \cdot 56$

$\begin{array}{cc}\text { Significance of epistasis } \$ & {[i]} \\ * * & -10.20 \\ * * * & -9.39 \\ * & -6.65 \\ \text { n.s. } & -1.68 \\ \text { n.s. } & -1.19 \\ * & -7.23 \\ \text { n.s. } & -4.54 \\ \text { n.s. } & 0.25\end{array}$

Growth Rate

Environmental value

$2 \cdot 11$

$2 \cdot 83$

3.31

3.34

$3 \cdot 62$

3.73

3.74

$4 \cdot 12$

Leaf length

Environmental value

13.94

$15 \cdot 00$

$15 \cdot 10$

$15 \cdot 12$

$15 \cdot 16$

$15 \cdot 50$

$15 \cdot 75$

16.52
Significance of epistasis

**.
n.s.
n.s.
n.s.
n.s.
n.s.
n.s.
***.

Significance of epistasis

$\begin{array}{lr}\text { n.s. } & -0.78 \\ \text { n.s. } & -0.01 \\ \text { n.s. } & -0.41 \\ \text { n.s. } & -0.59 \\ \text { n.s. } & 0.19 \\ \text { n.s. } & 0.51 \\ \text { n.s. } & 0.30 \\ \text { n.s. } \dagger & 0.54\end{array}$

$\dagger$ These two environments come closest to being significant with $P=0.08$ and $P=0.13$, respectively.

$\ddagger$ n.s., not significant.

* Probability $=0.05-0.01$. ** Probability $=0.01-0.001$. *** Probability $<0.001$. 
data. The estimates for three characters are presented. For two, growth rate and leaf length, a simple pattern emerges in which epistasis is most marked in the extreme environments, both high and low, and the estimates of [i] are negative in the lower environments and positive in the higher environments. When averaged over all the eight environments the epistatic components [i] are not significant for either character (Perkins and Jinks, 1973). Although, a similar pattern is discernible for final height, the third character presented in table 1 , the distribution of [i] values is truncated at the positive end, which corresponds with the high environmental values, relative to the other two characters.

The results of the analysis in the second set of data $(2 \times 42)$ of the character final height are summarised in table 2. They differ from those obtained from the $1 \times 5$ cross in showing the highest incidence of epistasis in the inter-

TABLE 2

Estimates of the environmental value and of the epistatic component, [i], and the significance of the epistasis for each of eight environments for final height in the cross $2 \times 42$

Environmental value
$\begin{gathered}105.4 \\ 113.6 \\ 113.7 \\ 116.2 \\ 117.6 \\ 123.9 \\ 124.9 \\ 131.6\end{gathered}$

$\begin{array}{cr}\text { Epistasis } \dagger & {[i]} \\ \text { n.s. } & -3 \cdot 82 \\ \text { n.s. } & 12 \cdot 10 \\ * * * & 35.77 \\ * * * & 16 \cdot 69 \\ \dagger & -10.04 \\ * & 8.38 \\ \text { n.s. } & 25.21 \\ * & 30.75\end{array}$

† See table 1 for probability levels.

mediate environments, in the irregularity of the magnitudes and sign of the values of $[i]$ and in the predominance of positive values of [i].

The characters we have examined so far have exhibited significant epistasis in some environments but not in others. Flowering time, which is available in both sets of data, differs in showing significant epistasis in all eight environments in both sets. The estimates of [i] for these flowering time data are listed in order of increasing environmental values in table 3. For the first set, which are taken from the $1 \times 5$ cross, all estimates of [i] are negative but they decrease regularly in magnitude with increasing environmental values. This trend is similar to that for final height in the same cross differing only in that the values of [i] for flowering time do not fall off sufficiently to be non-significant in the highest environments. For the second set, which are taken from the $2 \times 42$ cross, the estimates of [i] are again all negative but they show no obvious trend over the environmental range and in this respect they also resemble the final height data from the same cross. There is, however, a relationship between the values of [i] for flowering time and sowing date, the environmental variable. The larger values of [i] occur in the earliest and latest sowings (1, 2, 7 and 8 in table 3) and the smaller values in the intermediate sowings. There is, therefore, a relationship of the kind found for growth rate and leaf length in the cross $1 \times 5$ but to the physical measure of the environments rather than to the biological measure. 


\title{
5. The NATURE OF THE EPISTATIC COMPONENT
}

The genes controlling the various characters in the cross $1 \times 5$ are largely dispersed between the parental varieties (Jinks and Perkins, 1972; Perkins

TABLE 3

\begin{abstract}
Estimates of the environmental value and of the epistatic component, [i], for flowering time in each of eight environments for cross $1 \times 5$ and cross $2 \times 42$. For the latter cross the sowing sequence for the eight environments is also given
\end{abstract}

$1 \times 5$

$\begin{array}{cc}\text { Environmental value } & {[i]} \\ 71.69 & -5.34 \\ 72.44 & -5.83 \\ 74.69 & -4 \cdot 16 \\ 75.00 & -4.55 \\ 75.19 & -4.37 \\ 76.25 & -3.31 \\ 80.19 & -3.78 \\ 81.56 & -2.46\end{array}$

$2 \times 42$

Environmental value

$82 \cdot 80$

[i]

$83 \cdot 10$

$-30 \cdot 40$

$85 \cdot 80$

$-14.27$

$85 \cdot 85$

$-28.49$

87.05

$-35 \cdot 38$

89.01

$-17.57$

$92 \cdot 65$

$-18.84$

$96 \cdot 73$

$-37 \cdot 44$

$-22 \cdot 71$

\section{Sowing sequence}

6

4

7

8

3

5

2

and Jinks, 1973). The sign of $[i]$ relative to that of $[d]$ is, therefore, the opposite to that of the sum of $i$ over all pairs of interacting loci (Jinks and Jones, 1958; Mather and Jinks, 1971). Thus the consistent pattern which we have observed for all characters in this cross is interpretable as positive interactions at the lower end of the environmental range which are either reduced in magnitude or replaced by negative interactions at the upper end of the range.

The cross $2 \times 42$ has not been investigated prior to the experiments referred to in this paper and which were first described and analysed by Perkins and Jinks (1971). We lack, therefore, the detailed knowledge of the genetical control of final height and flowering time that would allow us to pursue further the epistatic component and its relationship with environmental change.

\section{The linearity OF the EPISTATIC COMPONENT}

In general we have found that epistasis occurs with the highest frequency and with the greatest magnitude at one or both extremes of the range of environments in which $\mathcal{N}$. rustica has been grown. This relationship can be examined further by regressing the estimates of the epistatic component [i] from each environment against the independently assessed value of the environment. This, in the standard terminology of Perkins and Jinks (1973), is regressing $g_{[i]\}}$ against $z_{j}$. The appropriate analyses have already been carried out for all the characters of the first set of data as part of an investigation into the validity of alternative assessments of the environmental values (Section 5, Perkins and Jinks, 1973). The conclusions reached which are 
relevant to the present discussion were that all the significant differences over environments in the magnitude of the epistatic components of flowering time and growth rate are linear functions of the independent environmental values. For final height and leaf length, however, there are significant $(P=0.001$ and $P=0.01-0.05)$ non-linear differences in the magnitude of the epistatic component.

Since the epistatic component [i] measures the interaction between the additive effects of genes at all pairs of loci, it is reasonable to examine whether or not the variation in this epistatic component over environments reflects the corresponding variation in the additive component, [d]. As estimated in this paper [i] and [d] are statistically independent and this procedure is equivalent to regressing estimates of $g_{[i] j}$ against independent estimates of $g_{d j}$ over the $j$ environments (Perkins and Jinks, 1973).

For both sets of data there is no evidence of a simple relationship between the epistatic and additive components for any characters.

\section{QUAlitative SUPPORT FROM EARLIER STUDIES}

The inbred varieties 1 and 5 and various generations derived from crosses between them have been grown in most seasons since 1946 (see Mather and Vines, 1952) and in 16 of them sufficient data on final height can be extracted

TABLE 4

The significance of the $\chi^{2}$ testing the goodness of fit of an additive-dominance model for each of 16 environments listed in order from the lowest to the highest environmental value

$\begin{array}{cc}\text { Environmental rank } & \text { Significance of } \chi^{2} \dagger \\ 1 & * * \\ 2 & * * \\ 3 & * * \\ 4 & \text { n.s. } \\ 5 & * * \\ 6 & \text { n.s. } \\ 7 & \text { n.s. } \\ 8 & * * \\ 9 & \text { n.s. } \\ 10 & \text { n.s. } \\ 11 & \text { n.s. } \\ 12 & * * \\ 13 & \text { n.s. } \\ 14 & \text { ** } \\ 15 & \text { ** } \\ 16 & \text { ** }\end{array}$

† See table 1 for probability levels.

to test the adequacy of an additive-dominance model for the family means and to rank the seasons on the basis of their environmental values. These data have already been described by Bucio Alanis (1966) and Bucio Alanis, Perkins and Jinks (1969) have already noted that epistasis is present in some seasons but that their contributions cancel out and become non-significant on averaging over all 16 seasons. The significances of the $\chi^{2}$ s testing the goodness of fit of a model which assumes epistasis to be absent are listed in rank order of the environmental values in table 4. To achieve some degree of 
uniformity and hence of sensitivity these analyses have been limited to the same six family means, namely, parents, $F_{1}, F_{2}, B_{1}$ and $B_{2}$, in each of the 16 seasons. Nevertheless, the data from the 16 seasons differ considerably in the overall sizes and structures of the experiment from which they were taken, in family size and in the number of replications. Quantitative comparisons are, therefore, not particularly rewarding because of the inevitable differences in sensitivity but qualitatively they show the now familiar concentration of the epistasis in both the upper and lower extreme environments (table 4). Four further experiments, two of which were replicated at two sites in 1965 (Jinks and Perkins, 1970) and two in 1967 and 1968, also fit this general picture. One of these, which would rank 11 th in the list in table 4, showed no epistasis. Of the other three, which would rank 17th, 18th and 19th, two showed significant epistasis. Thus over the 20 environments there is a qualitative relationship between the occurrence of epistasis and the ranking of the environments of the kind found in the $1 \times 5$ cross in the last section, although the environmental variables are of a quite different kind.

\section{Discussion}

In the $1 \times 5$ cross there is a clear dependence of the epistatic component of the family means in its occurrence, sign and magnitude on the environment. This may take the form of a linear relationship between the epistatic component and an independent measure of the environmental value, or it may be largely non-linear. But, where it can be analysed, it leads in all cases to large negative values of the epistatic component at the lower end of the environmental range which fall in magnitude as the environmental values increase. For some characters this fall is so marked that it becomes non-significant in the average environments and becomes large again but positive in sign at the higher end of the environmental range. Because the genes controlling these characters are mainly dispersed the sign of the interactions between pairs of loci is the reverse of that of the estimate of the epistatic component, [i]. We have a situation, therefore, in which the same character may display two or even three qualitatively distinct kinds of genetical architecture in different portions of the environmental range. These are the genetical architectures normally associated with characters which have been subjected to different types of selection (Mather, 1953; Breese and Mather, 1960; Kearsey and Kojima, 1967; Mather, 1967). For example, in the intermediate environments we find little or no evidence for epistasis for most characters. Either, therefore, the epistatic effect at every pair of loci is small or zero, or it is positive at some and negative at others and so cancels out on summing the effects over all pairs of loci. In either case the genetical architecture is that of a character with an intermediate optimum subject to stabilising selection. In extreme environments, on the other hand, the same characters have significant epistatis components which, for some characters, have opposite signs in the high and low ends of the environmental range. In these extreme environments they therefore display the genetical architecture of a character whose optimum is towards the extreme of its phenotypic distribution and subject to directional selection.

This change in genetical architecture with changing environment is that expected from the established relationship between the genetical architecture of a character and the type of selection to which it has been subjected provid- 
ing that the selection acting on a phenotype which deviates from the optimum as defined in an average environment is the same irrespective of whether this deviation is produced by an extreme genotype in an average environment or an average genotype in an extreme environment. Or to put it another way, for this relationship to hold for the present data we must assume that the same type of selection operates on a phenotypic deviation from the optimal phenotype whether this deviation is genetical or environmental in origin.

While much of the discussion has been concerned with those properties which are more or less common to all characters of the $1 \times 5$ cross, in the cross $2 \times 42$, which is the only other cross where comparable data are available, the distribution of epistasis over environments is quite different from that in $1 \times 5$. None of the simple relationships between the magnitude of the epistatic component and the environmental values found in the latter cross appear to apply to the $2 \times 42$ cross. Nevertheless, for flowering time in the cross $2 \times 42$, epistasis is most marked in the extreme environments as defined by the physical treatment which differentiates the environments, namely, date of sowing. The different pictures which emerge from the two crosses may, therefore, reflect the situation described by Perkins (1972) whereby the differences between the environmental values as measured biologically $\left(e_{j}\right.$ and $z_{j}$ ) could be accounted for by physical differences between the environments for families of the $1 \times 5$ cross but not for those of the $2 \times 12$ cross which is similar in many respects to the $2 \times 42$ cross.

\section{ReFERENCES}

BREESE, E. L., AND MATHER, K. 1960. The organisation of polygenic activity within a chromosome of Drosophila. II. Viability. Heredity, 15, 431-439.

Bucio ALANIs, L. 1966. Environmental and genotype-environmental components of variability. I. Inbred lines. Heredity, 21, 399-405.

bucio Alanis, L., PERKINs, JEAN M., AND JiNks, J. L. 1969. Environmental and genotypeenvironmental components of variability. V. Segregating generations. Heredity, 24, 115-127.

JINKS, J. L., AND JONES, MORLEY R. 1958. Estimation of the components of heterosis. Genetics, 43, 223-234.

JINKS, J. L., AND PERKINS, JEAN M. 1970. Environmental and genotype-environmental components of variability. VII. Simultaneous prediction across environments and generations. Heredity, 25, 475-480.

JINKs, J. L., AND PERKINs, JEAN M. 1972. Predicting the range of inbred lines. Heredity, 28, 399-403.

KEARSEY, M. J., AND KOJMM, KEN-ICHr. 1967. The genetic architecture of body weight and egg hatchability in Drosophila melanogaster. Genetics, 56, 24-37.

MATHER, K. 1953. The genetical structure of populations. S.E.B. Symposia, Evolution, VII, 66-95.

MATHER, K. 1967. Complementary and duplicate gene interaction in biometrical genetics. Heredity, 22, 97-103.

MATHER, K., AND Jnks, J. L. 1971. Biometrical Genetics, 2nd edn. Chapman and Hall, London.

MATHER, K., AND vines, A. 1952. The inheritance of height and flowering time in a cross of Nicotiana rustica. From Quantitative Inheritance (ed. E. C. Reeve and C. H. Waddington), pp. 49-80. H.M.S.O., London.

PERKINS, JEAN M. 1972. The principal component analysis of genotype-environmental interactions and physical measures of the environment. Heredity, 29, 51-70.

PERKINS, JEAN M., AND JINKs, J. L. 1971. Specificity of the interaction of genotypes with contrasting environments. Heredity, 26, 463-474.

PERKINS, JEAN M., AND JINKs, J. L. 1973. The assessment and specificity of environmental and genotype-environmental components of variability. Heredity, 30, (in press). 